\title{
Bir Eğri ve Üç Denklem Üzerine Bir Çalışma
}

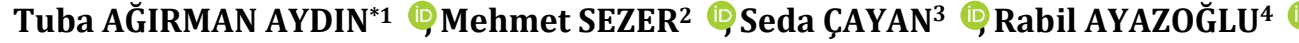 \\ 1,4Bayburt Üniversitesi, Eğitim Fakültesi, Matematik ve Fen Bilimleri Eğitimi Bölümü, 69000, Bayburt, Türkiye \\ 2,3 Celal Bayar Üniversitesi, Fen Edebiyat Fakültesi, Matematik Bölümü, 45000, Manisa, Türkiye
}

(Alınış / Received: 04.11.2020, Kabul / Accepted: 09.03.2021, Online Yayınlanma / Published Online: 15.08.2021)

\section{Anahtar Kelimeler}

Bernstein polinom yaklaşımı, Hermite polinom yaklaşımı, Sabit genișlikli eğri,

Frenet çatısı
Özet: Kinematik, mühendislik, sanat, cam dizayn ve geometri gibi birçok alanda çok özel bir yere sahip olan sabit genişlikli eğriler bu başlık altında özel olarak ele alınmıștır. Bu çalışmada sabit genişlikli eğrileri karakterize eden bir diferansiyel denklem sisteminin vasıtasıyla elde edilen üç diferansiyel denklem irdelenmiștir. Bu diferansiyel denklemler farklı değişkenlere bağlı üçüncü mertebeden, değişken katsayıl, homojen, lineer diferansiyel denklemlerdir. Bu denklemlerin farklı iki polinom yaklaşımı ile yaklaşık çözümleri hesaplanıp hata analizleri yapılmıştır. Elde edilen sonuçlar sayısal bir örnek üzerinden analiz edilerek en iyi sonuç veren yaklaşım metodu tespit edilmiştir. Bu denklemler farklı uzaylarda farklı çatılara göre farklı eğri tipleri için de bir karakterizasyon teşkil edebilmektedir. Dolayısıyla bu çalışma sadece bu eğri tipi için değil benzer denklemlerle ifade edilebilen tüm eğrilerin geometrisi için önem arz etmektedir.

\section{A Study on a Curve and Three Equations}

\section{Keywords}

Bernstein polynomial approximation, Hermite polynomial approximation,

Fixed-width curves, Frenet frame

\begin{abstract}
The fixed-width curves, which have a very special place in many fields such as kinematics, engineering, art, cam design and geometry, are specially discussed under this title. In this study, three differential equations obtained by means of a system of differential equations characterizing fixed-width curves are examined. These differential equations are third order, variable coefficient, homogeneous, linear differential equations based on different variables. Approximate solutions of these equations are calculated with two different polynomial approximations and error analysis is made for the solutions. Thus, the best approach method is determined for the most accurate result. Also, these equations can constitute a characterization for different types of curves according to different frames in different spaces. Therefore, this study is important not only for this curve type but also for the geometry of all curves that can be expressed with similar equations.
\end{abstract}

\section{Giriș}

Bir eğri üzerinde teğetlerin paralel ve zit yönlü olduğu iki nokta, "karşıt noktalar" olarak adlandırılır. $\mathrm{Bu}$ iki nokta arasındaki mesafe eğrinin genişliği olarak ifade edilir. Bir doğrultu ve bu doğrultuya dik, birine paralel bir çift destek doğrusu arasındaki mesafe de bir figürün seçilen doğrultuda genişliği olarak tanımlanır. Üzerindeki tüm karşıt noktalar arasındaki mesafe sabit ve birbirine eşit olan eğri sabit genişlikli eğri olarak tanımlanırken her doğrultuda aynı genişliğe sahip olan şekiller de sabit genişlikli şekiller olarak adlandırılır $[1,2]$.

$\mathrm{Bu}$ özel eğri ve şekiller özelliklerinin bir kısmı ile birlikte uzun süredir bilinmektedir. İlk olarak Euler onları daire şeklindeki eğriler için Latince "orbiforms" adı altında inceledi. Euler sınırları bir hiposikloidin evrimi olarak gösterilebilecek sabit genişlikli eğrilerle ilgilendi [3]. Sonrasında bir mühendis ve matematikçi olan Reuleaux, mühendislikteki kinematik üzerine yayınladı̆̆ kitabında sabit genişlikteki şekilleri de ele aldı. Bu kitapta, daireden sonra belki de en basit sabit genişlikli şekil olan ve bugün kendi adını taşıyan üçgeni tanımladı. Bu geometrik şekil zaten uzun zamandır biliniyordu ancak Reuleaux sabit genişlik özelliklerine ilk odaklanan kişi oldu [4]. Reuleaux üçgeni bir eşkenar üçgenden yola çıkılarak elde edilir, benzer mantıkla farklı düzgün çokgenler üzerine sabit genişlikli birçok şekil oluşturmak mümkündür.

Sabit genişlikli şekiller kendi yüzey alanından geçmeyen konveks şekillerdir. Onları farklı kılan özelliklerinden dolayı birçok geometrici bu eğri tipine ilgi göstermiştir $[1,5,6]$. Bilginin birçok 
dalında bu karakterizasyona sahip şekillerin kullanımını gözlemlemek mümkündür. Bu eğrilerin mühendislikte kamlar (mil dirsekleri, makine parçası), kare delik açan matkaplar ve döner, içten yanmalı motorlardaki pistonlar, rögar kapakları gibi birçok farklı uygulaması vardır. Eski sinema filmi projektörlerinin çoğunda kullanılan basit bir cam mekanizmasının bir parçası, özel bir sabit genişlikli eğridir [7, 8]. Su çarkları bu eğri tipini kapsayan sistem üzerine kurulmuştur. Kablosuz ağların lokalizasyon ve kapsama alanlarında kullanılan temel eğriler bu türdedir [9-11]. Sanatta, sabit genişlikli eğri figürlerinin önemli rol oynadı̆̆ birçok dekoratif uygulama vardır. Özellikle en yaygın bilinen sabit genişlikli şekil olan çember veya dairesel kesitlere sahip silindir birçok mekanizmada, mimaride, tasarımda kullanılmaktadır. Çemberin ve dairesel parçanın daha çok tercih edilmesini sağlayan, denklem ve uygulama anlamında erişilebilir olmasıdır. Oysaki çember dişında sabit genişlikli farklı eğrilerin kullanılmasının birtakım avantajlar ortaya koyduğu farklı çalışma alanlarında gözlemlenmiştir. Örneğin yan arakesiti Reuleaux üçgeni biçiminde olan bir karayolu tankerinin devrilme olasılığının yan arakesiti çember biçiminde olan tankerin devrilme olasılığından daha az olduğu yapılan hesaplamalarla ortaya konulmuștur [12]. Bunun gibi örnekler çoğaltılabilir. Burada daha avantajlı bir durumun tercih edilmemesinin gerekçesi olarak alternatife ilişkin bilginin yetersiz olması gösterilebilir. Bu noktada böyle bir açığı kapatmak adına farklı uzaylarda yapılmış birçok çalışma bulmak mümkündür $[13,14]$.

$\mathrm{Bu}$ makalede öncelikle diferansiyel denklem sistemi yoluyla bu eğrileri karakterize eden benzer yapıda üç farklı üçüncü mertebeden, değişken katsayılı, homojen, lineer diferansiyel denklem elde edilmiştir. Sonra bu diferansiyel denklemler için farklı iki yöntemle yaklaşık çözümler hesaplanarak hata analizleri yapılmıştır. Sayısal bir örnekle bu eğriler için en uygun yaklaşım metodu hata analizleri yardımıyla saptanmıştır. Sabit genişlikli eğri tipinin diferansiyel denklemlerle ifadesi her alanda rahatça kullanımına ortam hazırlayacaktır. Ayrıca aynı başlık altındaki farklı modellerin deneyimlenebilme imkanı, kullanımda daha yüksek verim sağlayabilecektir.

Bu çalışmada hesaplamalar Mathematica programı ile yapılmiştır.

\section{Materyal ve Metot}

$\mathrm{Bu}$ bölümde sabit genişlikli eğrileri karakterize eden üç farklı diferansiyel denklem elde edilerek bu denklemlerin yaklaşık çözümleri için iki farklı metot verilir.

\subsection{Sabit genişlikli uzay eğrileri}

$\gamma$ Öklid uzayında eğrilik ve torsiyonu sıfır olmayan $C^{3}$ sınıfından birim hızlı basit kapalı bir eğri ve eğri üzerindeki $\gamma(s)$ ve $\gamma^{*}$ karşıt iki nokta olsun. $\gamma$ eğrisinin vektör pozisyonu

$$
\gamma^{*}(s)=\gamma(s)+\lambda(s) \vec{t}+\mu(s) \vec{n}+\delta(s) \vec{b}
$$

ile ifade edilir, burada $\vec{t}, \vec{n}, \vec{b}$ Frenet çatı bileşenleridir [2]. Bu eşitlik s yay uzunluğu parametresine göre diferansiyellenerek ve Frenet formülleri kullanılarak

$$
\begin{aligned}
\frac{d \gamma^{*}}{d s}=\vec{t}^{*} \frac{d s^{*}}{d s}=( & \left.1+\frac{d \lambda}{d s}-\mu(s) \kappa\right) \vec{t} \\
& +\left(\lambda(s) \kappa+\frac{d \mu}{d s}-\delta(s) \tau\right) \vec{n} \\
& +\left(\frac{d \delta}{d s}+\mu(s) \tau\right) \vec{b}
\end{aligned}
$$

ifadesi elde edilir. Karşıt noktalarda $\vec{t}=-\vec{t}^{*}$ olduğundan

$$
\begin{gathered}
-\frac{d s^{*}}{d s}=1+\frac{d \lambda}{d s}-\mu(s) \kappa \\
\lambda(s) \kappa+\frac{d \mu}{d s}-\delta(s) \tau=0 \\
\frac{d \delta}{d s}+\mu(s) \tau
\end{gathered}
$$

bulunur. $\Delta \varphi$ kontengenez açısı olmak üzere $\gamma$ eğrisinin eğriliğinin $\quad \kappa=\lim \frac{\Delta \varphi}{\Delta s}=\frac{d \varphi}{d s} \quad$ olduğu bilinmektedir. Böylece (2) diferansiyel denklem sistemi

$$
\begin{aligned}
& \frac{d \lambda}{d \varphi}=\mu-f(\varphi) \\
& \frac{d \mu}{d \varphi}=\rho \tau \delta-\lambda \\
& \frac{d \delta}{d \varphi}=-\rho \tau \mu
\end{aligned}
$$

formunda yazılabilir. Burada $f(\varphi)=\rho+\rho^{*}$ ve $\rho=$ $\frac{1}{\kappa}, \rho^{*}=\frac{1}{\kappa^{*}}$ eğrinin $\gamma(s)$ ve $\gamma^{*}(s)$ noktalarında eğrilik yarıçapıdır. Diğer taraftan eğer $\gamma(s)$ ve $\gamma^{*}(s)$ karşıt noktaları arasındaki mesafe sabit ise $\left\|\gamma^{*}-\gamma\right\|^{2}=$ $\|d\|^{2}=\lambda^{2}+\mu^{2}+\delta^{2}=$ sabit yazılır. $\lambda^{2}+\mu^{2}+\delta^{2}=$ sabit eşitliği $\varphi$ ye göre diferansiyellenirse $\lambda \lambda^{\prime}+$ $\mu \mu^{\prime}+\delta \delta^{\prime}=0$ elde edilir. $\mathrm{Bu}$ eşitlik (3) sistemi yardımıyla $\lambda\left(\lambda^{\prime}-\mu\right)=0$ eşitliğine indirgenebilir. Burada iki temel durum mümkündür:

I. durum: $\lambda=0$,

II. durum: $\lambda^{\prime}-\mu=0$.

I. durumda (3.1) eşitliğinden $\mu=f(\varphi)$ elde edilir. $\lambda=0$ ve $\mu=f(\varphi)$ için (3) diferansiyel denklem sistemi

$$
\begin{aligned}
& \mu=f(\varphi) \\
& \frac{d \mu}{d \varphi}=\rho \tau \delta \\
& \frac{d \delta}{d \varphi}=-\rho \tau \mu
\end{aligned}
$$


biçimine dönüşür. $\delta$ ve türevleri yok edilerek elde edilen $\mu$ katsayısına bağlı ikinci mertebeden değişken katsayılı lineer denklemden A ve B integrasyon sabiti olmak üzere

$$
\mu=A \cos \int_{0}^{\varphi} \rho \tau d \varphi+B \sin \int_{0}^{\varphi} \rho \tau d \varphi=f(\varphi)
$$

elde edilir. $\mu$ ve türevleri yok edilerek elde edilen denklemden de $\delta$ aşağıdaki gibi hesaplanır:

$$
\delta=-A \sin \int_{0}^{\varphi} \rho \tau d \varphi+B \cos \int_{0}^{\varphi} \rho \tau d \varphi .
$$

$\mathrm{Bu}$ eşitlikler (1) denkleminde kullanılırsa, $\lambda=0$ için eğrinin integral karakterizasyonu aşağıdaki gibi olur:

$$
\begin{gathered}
\gamma^{*}=\gamma+\left[A \cos \int_{0}^{\varphi} \rho \tau d \varphi+B \sin \int_{0}^{\varphi} \rho \tau d \varphi\right] \vec{n}+ \\
{\left[-A \sin \int_{0}^{\varphi} \rho \tau d \varphi+B \cos \int_{0}^{\varphi} \rho \tau d \varphi\right] \vec{b} .}
\end{gathered}
$$

Ayrıca bu durumda eğrinin sabit genișlik değeri yani karşıt noktalar arasındaki mesafe $\|d\|=\sqrt{A^{2}+B^{2}}$ olarak elde edilir.

II. durumda $\lambda^{\prime}=\mu$ olacağından (3.1) eşitliğinden $f=0$ elde edilir. $f=0$ için $d$ mesafesi sabit olacağından $\gamma$ eğrisi sabit genişlikli olur. Öyleyse (3) sisteminde $f=0$ alınırsa sabit genişlikli eğrileri karakterize eden aşağıdaki diferansiyel denklem sistemi elde edilir:

$$
\begin{aligned}
& \frac{d \lambda}{d \varphi}=\mu \\
& \frac{d \mu}{d \varphi}=\rho \tau \delta-\lambda \\
& \frac{d \delta}{d \varphi}=-\rho \tau \mu
\end{aligned}
$$

(4) diferansiyel denklem sisteminden $\mu, \delta$ ve bunların türevleri yok edilirse aşağıdaki 3 . mertebeden değişken katsayll, homojen, lineer diferansiyel denklem elde edilir:

$$
\rho \tau \lambda^{\prime \prime \prime}-(\rho \tau)^{\prime} \lambda^{\prime \prime}+\left(\rho \tau+\rho^{3} \tau^{3}\right) \lambda^{\prime}-(\rho \tau)^{\prime} \lambda=0
$$

$\mathrm{Bu}$ Öklid uzayında sabit genişlikli eğrileri $\lambda$ katsayısına bağlı ifade eden diferansiyel denklemdir. Benzer biçimde (4) diferansiyel denklem sisteminden $\mu, \delta$ ve bunların türevleri yok edilerek aşağıdaki teorem yazılabilir.

Teorem 1: $\gamma$ Öklid uzayında eğrilik ve torsiyonu sıfır olmayan, birim hızlı, basit kapalı bir eğri olsun. $\gamma$ sabit genişlikli ise eğriyi $\mu$ katsayısına bağlı karakterize eden diferansiyel denklem aşağıdaki gibidir:

$$
\begin{aligned}
\frac{1}{(\rho \tau)^{\prime}} \mu^{\prime \prime \prime}+\left(\frac{1}{(\rho \tau)^{\prime}}\right)^{\prime} \mu^{\prime \prime}+\frac{1+(\rho \tau)^{2}}{(\rho \tau)^{\prime}} \mu^{\prime} \\
+\left[\left(\frac{1+(\rho \tau)^{2}}{(\rho \tau)^{\prime}}\right)^{\prime}+\rho \tau\right] \mu \\
=0 .
\end{aligned}
$$

Yine aynı düșünceyle (4) diferansiyel denklem sisteminden $\lambda, \mu$ ve bunların türevleri yok edilerek aşağıdaki teorem yazılabilir.

Teorem 2: $\gamma$ Öklid uzayında eğrilik ve torsiyonu sıfır olmayan, birim hızlı, basit kapalı bir eğri olsun. $\gamma$ sabit genişlikli ise eğriyi $\delta$ katsayısına bağlı karakterize eden diferansiyel denklem aşağıdaki gibidir:

$$
\begin{gathered}
\frac{1}{\rho \tau} \delta^{\prime \prime \prime}+2\left(\frac{1}{\rho \tau}\right)^{\prime} \delta^{\prime \prime}+\left[\left(\frac{1}{\rho \tau}\right)^{\prime \prime}+\frac{1+(\rho \tau)^{2}}{\rho \tau}\right] \delta^{\prime} \\
+(\rho \tau)^{\prime} \delta=0 .
\end{gathered}
$$

Öklid uzayında Frenet çatısına göre sabit genişlikli eğrilerin vektör pozisyonu (5), (6) ve (7) ile ifade edilebilir. Bunlar üçüncü mertebeden değişken katsayılı, homojen, lineer diferansiyel denklemlerdir. Böyle diferansiyel denklemler için nümerik çözüm elde edilemez ancak Legendre, Hermite, Taylor, Bernstein gibi farklı polinom yaklașımlarıyla geliștirilen matris sıralama yöntemleri kullanılarak yaklaşık çözüm elde edilebilir $[15,16,17]$. Bu çalışmada yaklaşık çözüm için Bernstein ve Hermite polinom yaklaşımı kullanılmıştır. Öncelikle, $k_{1}$ ve $k_{2}$ eğriliklerinin $s$ yay uzunluğu parametresine bağlı olduğu bu denklemler (5) için

$$
\begin{gathered}
P_{0}(s)=-(\rho \tau)^{\prime}, P_{1}(s)=\left(\rho \tau+\rho^{3} \tau^{3}\right), P_{2}(s) \\
=-(\rho \tau)^{\prime}, P_{3}(s)=\rho \tau
\end{gathered}
$$

(6) için

$$
\begin{aligned}
P_{0}(s)=\left(\frac{1+(\rho \tau)^{2}}{(\rho \tau)^{\prime}}\right)^{\prime}+\rho \tau, P_{1}(s) \\
=\frac{1+(\rho \tau)^{2}}{(\rho \tau)^{\prime}}, P_{2}(s) \\
=\left(\frac{1}{(\rho \tau)^{\prime}}\right)^{\prime}, P_{3}(s)=\frac{1}{(\rho \tau)^{\prime}}
\end{aligned}
$$

(7) için

$$
\begin{aligned}
P_{0}(s)=(\rho \tau)^{\prime}, P_{1} & (s) \\
& =\left(\frac{1}{\rho \tau}\right)^{\prime \prime}+\frac{1+(\rho \tau)^{2}}{\rho \tau}, P_{2}(s) \\
& =2\left(\frac{1}{\rho \tau}\right)^{\prime}, P_{3}(s)=\frac{1}{\rho \tau}
\end{aligned}
$$

olmak üzere genel biçimde

$$
\sum_{k=0}^{m} P_{k}(s) X^{(k)}(s)=0 \quad(m=3)
$$


şeklinde ifade edilebilir. Burada (5) için $X=\lambda$, (6) için $X=\mu$ ve (7) için $X=\delta$ olacağı açıtıtır.

\subsection{Bernstein polinom yaklaşımı metodu}

(8) genel denkleminin

$$
X(s) \cong X_{N}(s)=\sum_{n=0}^{N} a_{n} B_{n, N}(s) \quad(N \geq 3)
$$

şeklinde Bernstein polinomları formunda bir yaklaşık çözümü olduğu kabul edilsin. n. dereceden Bernstein polinomları $[0, R]$ aralığında aşağıdaki gibi tanımlanır:

$$
B_{n, N}(s)=\left(\begin{array}{l}
N \\
n
\end{array}\right) \frac{s^{n}(R-s)^{N-n}}{R^{N}}(n=0,1, \ldots, N) .
$$

Burada

$$
(R-s)^{N-n}=\sum_{i=0}^{N-n}\left(\begin{array}{c}
N-n \\
i
\end{array}\right)(-1)^{i} R^{N-n-i} s^{i}
$$

eşitliği kullanılırsa Bernstein taban polinomu için aşağıdaki eşitlik elde edilir:

$$
B_{n, N}(s)=\sum_{i=0}^{N-n}\left(\begin{array}{l}
N \\
n
\end{array}\right)\left(\begin{array}{c}
N-n \\
i
\end{array}\right) \frac{(-1)^{i}}{R^{n+i}} s^{n+i},
$$

R tanımlanan aralığın maksimum değeridir $[17,18]$. (9) yaklaşık çözümü ve türevi

$$
\begin{gathered}
\boldsymbol{B}_{N}(s)=\left[\begin{array}{llll}
\boldsymbol{B}_{0, N}(s) & \boldsymbol{B}_{1, N}(s) & \ldots & \boldsymbol{B}_{n, N}(s)
\end{array}\right], \\
\boldsymbol{A}=\left[\begin{array}{lllll}
a_{0} & a_{1} & \ldots & a_{N}
\end{array}\right]^{T}
\end{gathered}
$$

şeklinde tanımlanan matrisler kullanılarak

$$
\boldsymbol{X}(s)=\boldsymbol{B}_{N}(s) \boldsymbol{A}, \boldsymbol{X}^{(k)}(s)=\boldsymbol{B}_{N}^{(k)}(s) \boldsymbol{A}
$$

biçiminde ifade edilir. Bernstein taban polinomunun katsayıları

$$
\begin{gathered}
d_{i j}=\left\{\begin{array}{l}
\frac{(-1)^{j-i}}{R^{j}}\left(\begin{array}{c}
N \\
i
\end{array}\right)\left(\begin{array}{c}
N-i \\
j-i
\end{array}\right), \quad i \leq j \\
0, i>j
\end{array}\right. \\
\boldsymbol{D}=\left[\begin{array}{cccc}
d_{00} & d_{01} & \ldots & d_{0 N} \\
d_{10} & d_{11} & \ldots & d_{1 N} \\
\vdots & \vdots & \ddots & \vdots \\
d_{N 0} & d_{N 1} & \ldots & d_{N N}
\end{array}\right]
\end{gathered}
$$

tanımlaması kullanılarak

$$
\boldsymbol{D}=\left[\begin{array}{cccc}
1 & -\frac{3}{R} & \frac{3}{R^{2}} & -\frac{1}{R^{3}} \\
0 & \frac{3}{R} & -\frac{6}{R^{2}} & \frac{3}{R^{3}} \\
0 & 0 & \frac{3}{R^{2}} & -\frac{3}{R^{3}} \\
0 & 0 & 0 & \frac{1}{R^{3}}
\end{array}\right]
$$

matrisi ile ifade edilir. Dolayısıyla Bernstein taban polinomu, $S(s)=\left[\begin{array}{llll}1 & s & \cdots & s^{n}\end{array}\right]$ olmak üzere aşağıdaki matris eşitliği ile ifade edilir:

$$
\boldsymbol{B}_{N}(s)=\boldsymbol{S}(s) \boldsymbol{D}^{T}
$$

Diğer taraftan bu yaklaşık çözümün türevinin

$$
\boldsymbol{B}_{N}^{(k)}(s)=\boldsymbol{S}^{(k)}(s) \boldsymbol{D}^{T}
$$

şeklinde matris formunda ifadesi için $S$ matrisinin türevleri

$$
\begin{gathered}
\boldsymbol{S}^{\prime}(s)=\boldsymbol{S} \boldsymbol{B} \\
\boldsymbol{S}^{\prime \prime}(s)=\boldsymbol{S}^{\prime} \boldsymbol{B}=\boldsymbol{S} \boldsymbol{B}^{2} \\
\vdots \\
\boldsymbol{S}^{(k)}(s)=\boldsymbol{S}^{(k-1)} \boldsymbol{B}=\cdots=\boldsymbol{S} \boldsymbol{B}^{k}
\end{gathered}
$$

biçiminde tanımlanır. Burada B matrisi aşağıdaki gibidir:

$$
\boldsymbol{B}=\left[\begin{array}{ccccc}
0 & 1 & 0 & \cdots & 0 \\
0 & 0 & 2 & \cdots & 0 \\
\vdots & \vdots & \vdots & \ddots & \vdots \\
0 & 0 & 0 & \cdots & N \\
0 & 0 & 0 & \cdots & 0
\end{array}\right]
$$

Son olarak (11) ve (12) matris formlarının (10) da yerine konulmasıyla aşağıdaki matris bağıntıları elde edilir:

$$
\boldsymbol{X}(s)=\boldsymbol{S} \boldsymbol{D}^{T} \boldsymbol{A}, \boldsymbol{X}^{(k)}(s)=\boldsymbol{S} \boldsymbol{B}^{k} \boldsymbol{D}^{T} \boldsymbol{A} .
$$

Şimdi bu (13) eşitlikleri (8) de kullanılırsa genel formda

$$
\sum_{k=0}^{m} \boldsymbol{P}_{k}(s) \boldsymbol{S}(s) \boldsymbol{B}^{k} \boldsymbol{D}^{T} \boldsymbol{A}=\boldsymbol{G}
$$

matris denklemi elde edilir. Bu denklemde $s_{i}=a+$ $\frac{b-a}{N} i,(a \leq s \leq b)$ sıralama noktaları kullanılarak (8) eşitliği aşağıdaki gibi matris denklemlerinin bir sistemi olarak ifade edilir:

$$
\sum_{k=0}^{m} \boldsymbol{P}_{k}\left(s_{i}\right) \boldsymbol{S}\left(s_{i}\right) \boldsymbol{B}^{k} \boldsymbol{D}^{T} \boldsymbol{A}=\boldsymbol{G}\left(s_{i}\right),
$$

$(i=0,1, \ldots, N) . \mathrm{Bu}$ temel matris denklemi kapalı formda

$$
\sum_{k=0}^{m} \boldsymbol{P}_{k} \boldsymbol{S} \boldsymbol{B}^{k} \boldsymbol{D}^{T} \boldsymbol{A}=\boldsymbol{G}
$$

biçiminde yazılabilir. Bu bağıntı

$$
\begin{gathered}
\boldsymbol{W}_{B}=\sum_{k=0}^{m} \boldsymbol{P}_{k} \boldsymbol{S} \boldsymbol{B}^{k} \boldsymbol{D}^{T}, \\
\boldsymbol{W}_{B}=\left[w_{k h}\right], \quad(k, h=0, \ldots, m)
\end{gathered}
$$

olmak üzere 


$$
\boldsymbol{W}_{B} \boldsymbol{A}=\boldsymbol{G} \text { veya }\left[\boldsymbol{W}_{B} ; \boldsymbol{G}\right]=\boldsymbol{A}
$$

biçiminde kısaltılabilir. Diğer taraftan koşulların matris denklemi

$$
\boldsymbol{S}(0) \boldsymbol{B}^{k} \boldsymbol{D}^{T} \boldsymbol{A}=\left[\alpha_{k}\right], \quad(k=0,1 \ldots, N-1)
$$

eşitliği yardımıyla matris formunda

$$
\boldsymbol{U}_{k} \boldsymbol{A}=\left[\alpha_{k}\right] \text { veya }\left[\boldsymbol{U}_{k} ; \alpha_{k}\right](k=0,1, \ldots, N-1)
$$

biçiminde ifade edilir. (17) satır matrisleri (16) matrisinin herhangi (N) satırı ile değiştirilerek, $\left[\widetilde{\boldsymbol{W}_{B}} ; \widetilde{\boldsymbol{G}}\right]$ arttırılmış matrisi hesaplanır ve $\boldsymbol{A}=$ $\left(\widetilde{\boldsymbol{W}_{B}}\right)^{-1} \widetilde{\boldsymbol{G}}$ olmak üzere bilinmeyenler matrisi bulunur. Son olarak bu $a_{k}$ değerleri (9) denkleminde yerine yazılırsa (8) denkleminin Bernstein polinom yaklaşımı ile yaklaşık çözümü elde edilir.

Özel olarak kesme sinırı $N=3$ alınıp $0 \leq s \leq 2 \pi$ aralığında (8) denkleminin (9) formunda yaklaşık çözümü elde edilecektir. Öncelikle $N=3$ için yaklaşık çözüm ve türevleri

$$
\begin{aligned}
\boldsymbol{B}_{3}(s)= & {\left[\begin{array}{llll}
\boldsymbol{B}_{0,3}(s) & \boldsymbol{B}_{1,3}(s) & \cdots & \boldsymbol{B}_{3,3}(s)
\end{array}\right] } \\
& \boldsymbol{A}=\left[\begin{array}{llll}
a_{0} & a_{1} & a_{2} & a_{3}
\end{array}\right]^{T}
\end{aligned}
$$

olmak üzere

$$
\boldsymbol{X}(s)=\boldsymbol{B}_{3}(s) \boldsymbol{A} \text { ve } \boldsymbol{X}^{(k)}(s)=\boldsymbol{B}_{3}^{(k)}(s) \boldsymbol{A}
$$

şeklinde elde edilir. Burada $[0,2 \pi]$ aralığında

$$
\begin{gathered}
\boldsymbol{D}=\left[\begin{array}{cccc}
1 & -\frac{3}{2 \pi} & \frac{3}{4 \pi^{2}} & -\frac{1}{8 \pi^{3}} \\
0 & \frac{3}{2 \pi} & -\frac{3}{2 \pi^{2}} & \frac{3}{8 \pi^{3}} \\
0 & 0 & \frac{3}{4 \pi^{2}} & -\frac{3}{8 \pi^{3}} \\
0 & 0 & 0 & \frac{1}{8 \pi^{3}}
\end{array}\right], \\
\boldsymbol{B}=\left[\begin{array}{llll}
0 & 1 & 0 & 0 \\
0 & 0 & 2 & 0 \\
0 & 0 & 0 & 3 \\
0 & 0 & 0 & 0
\end{array}\right], \boldsymbol{S}(s)=\left[\begin{array}{llll}
1 & S & s^{2} & S^{3}
\end{array}\right]
\end{gathered}
$$

matrisleri kullanılarak

$$
\boldsymbol{B}_{3}(s)=\boldsymbol{S}(s) \boldsymbol{D}^{T}, \boldsymbol{B}_{3}^{(k)}(s)=\boldsymbol{S}^{(k)}(s) \boldsymbol{D}^{T}
$$

eşitlikleri yazılır ve aşağıdaki matris bağıntıları elde edilir:

$$
\begin{gathered}
\boldsymbol{X}(s)=\boldsymbol{S} \boldsymbol{D}^{T} \boldsymbol{A}, \boldsymbol{X}^{\prime}(s)=\boldsymbol{S} \boldsymbol{B} \boldsymbol{D}^{T} \boldsymbol{A} \\
\boldsymbol{X}^{\prime \prime}(s)=\boldsymbol{S} \boldsymbol{B}^{2} \boldsymbol{D}^{T} \boldsymbol{A}, \boldsymbol{X}^{\prime \prime \prime}(s)=\boldsymbol{S} \boldsymbol{B}^{3} \boldsymbol{D}^{T} \boldsymbol{A} .
\end{gathered}
$$

Bu eşitlikler (8) de kullanılırsa

$$
\sum_{k=0}^{m=3} \boldsymbol{P}_{k}(s) \boldsymbol{S}(s) \boldsymbol{B}^{k} \boldsymbol{D}^{T} \boldsymbol{A}=\boldsymbol{G}
$$

matris denklemi elde edilir. Bu denklemde

$$
s_{0}=0, \quad s_{1}=\frac{2 \pi}{3}, \quad s_{2}=\frac{4 \pi}{3}, \quad s_{3}=2 \pi
$$

sıralama noktaları kullanılırsa aşağıdaki matrislerin oluşacağı açıktır:

$$
\begin{gathered}
\boldsymbol{P}_{k}=\left[\begin{array}{cccc}
P_{k}(0) & 0 & 0 & 0 \\
0 & P_{k}\left(\frac{2 \pi}{3}\right) & 0 & 0 \\
0 & 0 & P_{k}\left(\frac{4 \pi}{3}\right) & 0 \\
0 & 0 & 0 & P_{k}(2 \pi)
\end{array}\right], \\
\boldsymbol{S}=\left[\begin{array}{cccc}
1 & 0 & 0 & 0 \\
1 & \left(\frac{2 \pi}{3}\right) & \left(\frac{2 \pi}{3}\right)^{2} & \left(\frac{2 \pi}{3}\right)^{3} \\
1 & \left(\frac{4 \pi}{3}\right) & \left(\frac{4 \pi}{3}\right)^{2} & \left(\frac{4 \pi}{3}\right)^{3} \\
1 & (2 \pi) & (2 \pi)^{2} & (2 \pi)^{3}
\end{array}\right], \boldsymbol{G}=\left[\begin{array}{l}
0 \\
0 \\
0 \\
0
\end{array}\right] .
\end{gathered}
$$

Öyleyse (8) eşitliği $m=3$ için aşağıdaki gibi matris denklemlerinin bir sistemi olarak ifade edilir:

$$
\sum_{k=0}^{3} \boldsymbol{P}_{k}\left(s_{i}\right) \boldsymbol{S}\left(s_{i}\right) \boldsymbol{B}^{k} \boldsymbol{D}^{T} \boldsymbol{A}=\boldsymbol{G}\left(s_{i}\right),
$$

$(i=0,1, \ldots, 3)$. Bu temel matris denklemi kapalı formda

$$
\sum_{k=0}^{3} \boldsymbol{P}_{k} \boldsymbol{S} \boldsymbol{B}^{k} \boldsymbol{D}^{T} \boldsymbol{A}=\boldsymbol{G}
$$

biçiminde yazılabilir. Bu bağıntı

$$
\begin{gathered}
\boldsymbol{W}_{B}=\sum_{k=0}^{3} \boldsymbol{P}_{k} \boldsymbol{S} \boldsymbol{B}^{k} \boldsymbol{D}^{T}, \\
\boldsymbol{W}_{B}=\left[w_{k h}\right], \quad(k, h=0, \ldots, 3)
\end{gathered}
$$

olmak üzere

$$
\boldsymbol{W}_{B} \boldsymbol{A}=\boldsymbol{G} \text { veya }\left[\boldsymbol{W}_{B} ; \boldsymbol{G}\right]=\boldsymbol{A}
$$

biçiminde kısaltılabilir. Diğer taraftan koşullar için matris formu

$$
\begin{gathered}
\boldsymbol{U}_{0}=\left[\begin{array}{llll}
1 & 0 & 0 & 0
\end{array}\right] \\
\boldsymbol{U}_{1}=\left[\begin{array}{llll}
-\frac{3}{2 \pi} & \frac{3}{2 \pi} & 0 & 0
\end{array}\right] \\
\boldsymbol{U}_{2}=\left[\begin{array}{llll}
\frac{3}{2 \pi^{2}} & -\frac{3}{\pi^{2}} & \frac{3}{2 \pi^{2}} & 0
\end{array}\right]
\end{gathered}
$$

olmak üzere

$$
\boldsymbol{U}_{k} \boldsymbol{A}=\left[\alpha_{k}\right] \text { veya }\left[\boldsymbol{U}_{k} ; \alpha_{k}\right] \quad(k=0,1,2)
$$

biçiminde elde edilir. $\mathrm{Bu}$ satır matrisleri $\boldsymbol{W}_{B}$ matrisinin herhangi 3 satırı ile değiştirilerek, $\left[\widetilde{\boldsymbol{W}_{B}} ; \widetilde{\boldsymbol{G}}\right]$ arttırılmış matrisi aşağıdaki gibi elde edilir: 


$$
\left[\begin{array}{cccccc}
w_{00} & w_{01} & w_{02} & w_{03} & ; & g(0) \\
u_{00} & u_{01} & u_{02} & u_{03} & ; & \alpha_{0} \\
u_{10} & u_{11} & u_{12} & u_{13} & ; & \alpha_{1} \\
u_{20} & u_{21} & u_{22} & u_{23} & ; & \alpha_{2}
\end{array}\right]
$$

burada $w_{i j}(i=0 j=0, \ldots, 3)$ değerleri aşağıdaki gibi hesaplanir:

$$
\begin{gathered}
w_{00}=P_{0}(0)-\frac{3}{2 \pi} P_{1}(0)+\frac{3}{2 \pi^{2}} P_{2}(0) \\
-\frac{3}{4 \pi^{3}} P_{3}(0), \\
w_{01}=\frac{3}{2 \pi} P_{1}(0)-\frac{3}{\pi^{2}} P_{2}(0)+\frac{9}{4 \pi^{3}} P_{3}(0), \\
w_{02}=\frac{3}{2 \pi^{2}} P_{2}(0)-\frac{9}{4 \pi^{3}} P_{3}(0), \\
w_{03}=\frac{3}{4 \pi^{3}} P_{3}(0) .
\end{gathered}
$$

Son olarak $\left(\widetilde{\boldsymbol{W}_{B}}\right)^{-1}$ matrisi

$$
\left[\begin{array}{cccc}
0 & 1 & 0 & 0 \\
0 & 1 & \frac{2 \pi}{3} & 0 \\
0 & 1 & \frac{4 \pi}{3} & \frac{2 \pi^{2}}{3} \\
\frac{1}{w_{03}} & -\frac{w_{00}+w_{01}+w_{02}}{w_{03}} & -\frac{2 \pi\left(w_{01}+2 w_{02}\right)}{3 w_{03}} & -\frac{2 \pi^{2} w_{02}}{3 w_{03}}
\end{array}\right]
$$

şeklinde hesaplanır ve $\boldsymbol{A}=\left(\widetilde{\boldsymbol{W}}_{B}\right)^{-1} \widetilde{\boldsymbol{G}}$ olmak üzere bilinmeyenler matrisi $\mathbf{A}$ için $a_{0}, a_{1}, \ldots, a_{3}$ aşağıdaki gibi bulunur:

$$
\begin{gathered}
a_{0}=\alpha_{0} \\
a_{1}=\alpha_{0}+\frac{2 \pi}{3} \alpha_{1} \\
a_{2}=\alpha_{0}+\left(\frac{4 \pi}{3}\right) \alpha_{1}+\left(\frac{2 \pi^{2}}{3}\right) \alpha_{2} \\
a_{3}=\left(-\frac{w_{00}+w_{01}+w_{02}}{w_{03}}\right) \alpha_{0} \\
+\frac{2 \pi\left(w_{01}+2 w_{02}\right)}{3 w_{03}} \alpha_{1} \\
-\left(\frac{2 \pi^{2} w_{02}}{3 w_{03}}\right) \alpha_{2} .
\end{gathered}
$$

Eğer bu $a_{k}$ bilinmeyenleri (9) denkleminde yerine yazılırsa (8) denkleminin Bernstein polinom yaklaşımı ile yaklaşık çözümü

$$
\begin{aligned}
X(s)=a_{0}+\frac{3}{2 \pi} & \left(a_{1}-a_{0}\right) s \\
& +\frac{3}{4 \pi^{2}}\left(a_{0}-2 a_{1}+a_{2}\right) s^{2} \\
& +\frac{1}{8 \pi^{3}}\left(-a_{0}+3 a_{1}-3 a_{2}\right. \\
& \left.+a_{3}\right) s^{3}
\end{aligned}
$$

şeklinde elde edilir. Bu Öklid uzayında Frenet çatısına göre sabit genişlikli eğrileri temsil eden (5), (6) ve (7) diferansiyel denklemleri için Bernstein polinom yaklaşımıyla bir çözümdür. Böylece eğriyi belirleyen $\lambda, \mu, \delta$ katsayı fonksiyonları elde edilmiş olur.

\subsection{Hermite Polinom Yaklaşımı}

(8) genel denkleminin

$$
X_{N}(s)=\sum_{n=0}^{N} a_{n} H_{n}(s) \quad(N \geq 3)
$$

şeklinde Hermite polinomları formunda bir yaklaşık çözümü olduğu kabul edilsin. Burada $a_{n}$ bilinmeyen Hermite katsayılarıdır ve Hermite polinomları aşağıdaki gibi tanımlanır [2]:

$$
H_{n}(s)=\sum_{k=0}^{\llbracket n / 2 \rrbracket}(-1)^{k} \frac{n !}{(n-2 k) ! k !} 2^{n-2 k} s^{n-2 k} .
$$

Öncelikle (18) yaklaşık çözümünden hareket edilerek çözüm ve denklem matris formunda elde edilsin. Bunun için (18) ile ifade edilen Hermite polinom çözümü ve türevleri,

$$
\begin{gathered}
H^{(k)}(s)=\left[\begin{array}{llll}
H_{0}^{(k)}(s) & H_{1}^{(k)}(s) & \cdots & H_{N}^{(k)}(s)
\end{array}\right], \\
H(s)=\left[\begin{array}{lllll}
H_{0}(s) & H_{1}(s) & \cdots & H_{N}(s)
\end{array}\right], \\
A=\left[\begin{array}{lllll}
a_{0} & a_{1} & \cdots & a_{N}
\end{array}\right]^{T}
\end{gathered}
$$

olarak tanımlanan matrisler kullanılarak

$$
\boldsymbol{X}(s)=\boldsymbol{H}(s) \boldsymbol{A} \text { ve } \boldsymbol{X}^{(k)}(s)=\boldsymbol{H}^{(k)}(\boldsymbol{s}) \boldsymbol{A}
$$

şeklinde matris formunda yazılabilir. Ayrıca $\boldsymbol{H}(s)$ ve türevi matris formunda

$$
\boldsymbol{H}(s)=\boldsymbol{S}(s) \boldsymbol{F} \text { ve } \boldsymbol{H}^{(k)}(\boldsymbol{s})=\boldsymbol{S}^{(k)}(s) \boldsymbol{F}
$$

șeklinde ifade edilir. Bu durumda

$$
\boldsymbol{X}(s)=\boldsymbol{S}(s) \boldsymbol{F} \boldsymbol{A} \text { ve } \boldsymbol{X}^{(k)}(s)=\boldsymbol{S}(s) \boldsymbol{B}^{k} \boldsymbol{F} \boldsymbol{A}
$$

eșitlikleri elde edilir. Burada

$$
\mathbf{F}=\left[\begin{array}{cccccc}
\frac{(-1)^{0} 0 ! 2^{0}}{0 ! 0 !} & 0 & \frac{(-1)^{1} 2 ! 2^{0}}{1 ! 0 !} & \cdots & \frac{(-1)^{\frac{N}{2}} N ! 2^{0}}{\left(\frac{N}{2}\right) ! 0 !} & 0 \\
0 & \frac{(-1)^{0} 1 ! 2^{1}}{0 ! 1 !} & 0 & \cdots & 0 & \frac{(-1)^{\frac{N-1}{2}} N ! 2^{1}}{\left(\frac{N-1}{2}\right) ! 1 !} \\
0 & 0 & \frac{(-1)^{0} 2 ! 2^{2}}{0 ! 2 !} & \cdots & \frac{(-1)^{\frac{N}{2}-1} N ! 2^{2}}{\left(\frac{N}{2}-1\right) ! 2 !} & 0 \\
\vdots & \vdots & \vdots & \ddots & \vdots & \vdots \\
0 & 0 & 0 & \cdots & \frac{(-1)^{0} N ! 2^{N}}{0 ! N !} & \frac{(-1)^{0} N ! 2^{N}}{0 ! N !} \\
N \text { çift } &
\end{array}\right]
$$

şeklinde tanımlanır. (20) eşitlikleri (8) de kullanılırsa aşağıdaki matris denklemi elde edilir:

$$
\sum_{k=0}^{N} \boldsymbol{P}_{k}(s) \boldsymbol{S}(s) \boldsymbol{B}^{k} \boldsymbol{F} \boldsymbol{A}=\boldsymbol{G}
$$


Bu temel matris denklemi kısaca

$$
\sum_{k=0}^{N} \boldsymbol{P}_{k} \boldsymbol{S} \boldsymbol{B}^{k} \boldsymbol{F} \boldsymbol{A}=\boldsymbol{G}
$$

biçiminde yazılabilir. Böylece (8) e karşılık gelen denklem kapalı matris formunda (22) biçiminde ifade edilmiş olur. Bu bağıntı

$$
\begin{gathered}
\boldsymbol{W}_{H}=\sum_{k=0}^{N} \boldsymbol{P}_{k} \boldsymbol{S} \boldsymbol{B}^{k} \boldsymbol{F}, \\
\boldsymbol{W}_{H}=\left[w_{k h}\right], \quad(k, h=0, \ldots, N)
\end{gathered}
$$

olmak üzere

$$
\boldsymbol{W}_{H} \boldsymbol{A}=\boldsymbol{G} \text { veya }\left[\boldsymbol{W}_{H} ; \boldsymbol{G}\right]=\boldsymbol{A}
$$

biçiminde kısaltılabilir. Diğer taraftan koşulların matris denklemi

$$
\boldsymbol{U}_{k}=\boldsymbol{S}(0) \boldsymbol{B}^{k} \boldsymbol{F} \boldsymbol{A}=\left[\alpha_{k}\right], \quad(k=0,1, \ldots, N-1)
$$

olmak üzere

$$
\boldsymbol{U}_{k} \boldsymbol{A}=\left[\alpha_{k}\right] \text { veya }\left[U_{k} ; \alpha_{k}\right]
$$

biçiminde elde edilir. (24) satır matrisleri (23) matrisinin herhangi 3 satırı ile değiștirilerek,

$$
\boldsymbol{A}=\left(\widetilde{\boldsymbol{W}_{H}}\right)^{-1} \widetilde{\boldsymbol{G}}
$$

olmak üzere bilinmeyenler matrisi bulunur.

Özel olarak kesme sınırı $N=3$ alınarak (8) denkleminin bu defa (18) formunda yaklaşık çözümü elde edilecektir. Bu kesme sınırı için $0 \leq s \leq 2 \pi$ aralığında sıralama noktaları yine $0 \leq s_{0}<\cdots<s_{3} \leq$ $2 \pi$ şeklinde olur. $X_{n}(s)$, (8) denkleminin bir çözümü olduğundan sıralama noktalarında (8) denklemini sağlar. Öyleyse $X_{n}(s)$ yaklaşık çözümü $s_{i}$ sıralama noktalarıyla birlikte (8) denkleminde yerine yazılırsa $a_{0}, a_{1}, \ldots, a_{3}$ katsayılarını içeren bir lineer denklem sistemi elde edilir. Bu sistem kullanılarak denklemin her bir terimi matris formunda ifade edilebilir ve matris sıralama yöntemi kullanılarak çözüm elde edilir. Bu durumda (21) ifadesinde

$$
s_{0}=0, \quad s_{1}=\frac{2 \pi}{3}, \quad s_{2}=\frac{4 \pi}{3}, \quad s_{3}=2 \pi
$$

sıralama noktaları kullanılırsa aşağıdaki matrislerin oluşacağı açıktır:

$$
\boldsymbol{P}_{k}=\left[\begin{array}{cccc}
P_{k}(0) & 0 & 0 & 0 \\
0 & P_{k}\left(\frac{2 \pi}{3}\right) & 0 & 0 \\
0 & 0 & P_{k}\left(\frac{4 \pi}{3}\right) & 0 \\
0 & 0 & 0 & P_{k}(2 \pi)
\end{array}\right],
$$

$$
\boldsymbol{S}=\left[\begin{array}{cccc}
1 & 0 & 0 & 0 \\
1 & \left(\frac{2 \pi}{3}\right) & \left(\frac{2 \pi}{3}\right)^{2} & \left(\frac{2 \pi}{3}\right)^{3} \\
1 & \left(\frac{4 \pi}{3}\right) & \left(\frac{4 \pi}{3}\right)^{2} & \left(\frac{4 \pi}{3}\right)^{3} \\
1 & (2 \pi) & (2 \pi)^{2} & (2 \pi)^{3}
\end{array}\right], \boldsymbol{G}\left(s_{i}\right)=\left[\begin{array}{l}
0 \\
0 \\
0 \\
0
\end{array}\right] .
$$

Ayrıca N=3 için

$$
\boldsymbol{F}=\left[\begin{array}{cccc}
1 & 0 & -2 & 0 \\
0 & 2 & 0 & -12 \\
0 & 0 & 4 & 0 \\
0 & 0 & 0 & 8
\end{array}\right]
$$

olarak hesaplanır. Öyleyse (8) eşitliği aşağıdaki gibi matris denklemlerinin bir sistemi olarak ifade edilir:

$$
\sum_{k=0}^{3} \boldsymbol{P}_{k}\left(s_{i}\right) \boldsymbol{S}\left(s_{i}\right) \boldsymbol{B}^{k} \boldsymbol{F} \boldsymbol{A}=\boldsymbol{G}\left(s_{i}\right)
$$

$(i=0,1, \ldots, 3)$. Bu temel matris denklemi

$$
\begin{gathered}
\boldsymbol{W}_{H}=\sum_{k=0}^{3} \boldsymbol{P}_{k} \boldsymbol{S} \boldsymbol{B}^{k} \boldsymbol{F}, \\
\boldsymbol{W}_{H}=\left[w_{k h}\right], \quad(k, h=0, \ldots, 3)
\end{gathered}
$$

olmak üzere

$$
\boldsymbol{W}_{H} \boldsymbol{A}=\boldsymbol{G} \text { veya }\left[\boldsymbol{W}_{H} ; \boldsymbol{G}\right]=\boldsymbol{A}
$$

biçiminde kısaltılabilir. Diğer taraftan koşulların matris denklemi

$$
\begin{gathered}
\boldsymbol{U}_{0}=\boldsymbol{S}(0) \boldsymbol{F}=\left[\begin{array}{llll}
1 & 0 & -2 & 0
\end{array}\right] \\
\boldsymbol{U}_{1}=\boldsymbol{S}(0) \boldsymbol{B} \boldsymbol{F}=\left[\begin{array}{llll}
0 & 2 & 0 & -12
\end{array}\right] \\
\boldsymbol{U}_{2}=\boldsymbol{S}(0) \boldsymbol{B}^{2} \boldsymbol{F}=\left[\begin{array}{llll}
0 & 0 & 8 & 0
\end{array}\right]
\end{gathered}
$$

olmak üzere

$$
\boldsymbol{U}_{k} \boldsymbol{A}=\left[\alpha_{k}\right] \text { veya }\left[U_{k} ; \alpha_{k}\right] \quad(k=0,1,2)
$$

biçiminde elde edilir. (26) satır matrisleri (25) matrisinin herhangi 3 satırı ile değiştirilerek, $\left[\widetilde{\boldsymbol{W}_{H}} ; \widetilde{\boldsymbol{G}}\right]$ matrisi aşağıdaki gibi elde edilir:

$$
\left[\begin{array}{llllll}
w_{00} & w_{01} & w_{02} & w_{03} & ; & g(0) \\
u_{00} & u_{01} & u_{02} & u_{03} & ; & \alpha_{0} \\
u_{10} & u_{11} & u_{12} & u_{13} & ; & \alpha_{1} \\
u_{20} & u_{21} & u_{22} & u_{23} & ; & \alpha_{2}
\end{array}\right]
$$

burada $w_{i j}(i=0 j=0, \ldots, 3)$ değerleri aşağıdaki gibi hesaplanır:

$$
\begin{gathered}
w_{00}=P_{0}(0), w_{01}=2 P_{1}(0), \\
w_{02}=-2 P_{0}(0)+8 P_{2}(0), \\
w_{03}=-12 P_{1}(0)+48 P_{3}(0)
\end{gathered}
$$

Son olarak $\left(\widetilde{\boldsymbol{W}}_{H}\right)^{-1}$ matrisi 


$$
\left[\begin{array}{cccc}
0 & 1 & 0 & \frac{1}{4} \\
\frac{1}{8 P_{3}(0)} & -\frac{P_{0}(0)}{8 P_{3}(0)} & -\frac{P_{1}(0)-4 P_{3}(0)}{8 P_{3}(0)} & -\frac{P_{2}(0)}{8 P_{3}(0)} \\
0 & 0 & 0 & \frac{1}{8} \\
\frac{1}{48 P_{3}(0)} & -\frac{P_{0}(0)}{48 P_{3}(0)} & -\frac{P_{1}(0)}{48 P_{3}(0)} & -\frac{P_{2}(0)}{48 P_{3}(0)}
\end{array}\right]
$$

şeklinde hesaplanır. $\boldsymbol{A}=\left(\widetilde{\boldsymbol{W}_{H}}\right)^{-1} \widetilde{\boldsymbol{G}}$ olmak üzere bilinmeyenler matrisi bulunur. Böylece bilinmeyenler matrisi $\mathbf{A}$ için $a_{0}, a_{1}, \ldots, a_{3}$ aşağıdaki gibi elde edilir:

$$
\begin{gathered}
a_{0}=\alpha_{0}+\frac{1}{4} \alpha_{2} \\
a_{1}=-\frac{P_{0}(0)}{8 P_{3}(0)} \alpha_{0}-\frac{P_{1}(0)-4 P_{3}(0)}{8 P_{3}(0)} \alpha_{1} \\
-\frac{P_{2}(0)}{8 P_{3}(0)} \alpha_{2} \\
a_{2}=\frac{1}{8} \alpha_{2} \\
a_{3}=-\frac{P_{0}(0)}{48 P_{3}(0)} \alpha_{0}-\frac{P_{1}(0)}{48 P_{3}(0)} \alpha_{1} \\
-\frac{P_{2}(0)}{48 P_{3}(0)} \alpha_{2} .
\end{gathered}
$$

Eğer bu $a_{k}$ bilinmeyenleri (18) denkleminde yerine yazılırsa (8) denkleminin Hermite polinom yaklaşımı ile yaklaşık çözümü aşağıdaki gibi bulunur:

$$
\begin{gathered}
X(s)=a_{0}+2 s a_{1}+\left(4 s^{2}-4\right) a_{2}+\left(8 s^{3}-\right. \\
12 s) a_{3} .
\end{gathered}
$$

Bu Öklid uzayında Frenet çatısına göre sabit genişlikli eğrileri temsil eden (5), (6) ve (7) diferansiyel denklemleri için Hermite polinom yaklaşımıyla bir çözümdür. Böylece eğriyi belirleyen $\lambda, \mu, \delta$ katsayı fonksiyonları elde edilmiş olur.

\subsection{Rezidüel Hata Analizi}

(9) denklemi ile verilen kesilmiş Bernstein serisi ve (18) denklemi ile verilen kesilmiş Hermite serisi, (8) denkleminin yaklaşık çözümleri olduğundan elde edilen yaklaşık çözümler ve çözümlerin türevleri (8) denkleminde yerine konulduğunda denklem yaklaşık olarak sağlanmalıdır. $\mathrm{Bu}$ sebeple $\rho(s) \tau(s)=\chi(s)$ olmak üzere, $0 \leq s_{i} \leq 2 \pi(i=0,1, \ldots, N)$ aralığında

$$
\begin{aligned}
& R \lambda_{N}\left(s_{i}\right)=\mid \chi\left(s_{i}\right) \lambda^{\prime \prime \prime}\left(s_{i}\right)-\chi^{\prime}\left(s_{i}\right) \lambda^{\prime \prime}\left(s_{i}\right) \\
& +\left(\chi\left(s_{i}\right)+\chi^{3}\left(s_{i}\right)\right) \lambda^{\prime}\left(s_{i}\right) \\
& -\chi^{\prime}\left(s_{i}\right) \lambda\left(s_{i}\right) \mid \cong 0 \\
& R \mu_{N}\left(s_{i}\right)=\mid \frac{1}{\chi^{\prime}\left(s_{i}\right)} \mu^{\prime \prime \prime}\left(s_{i}\right)+\left(\frac{1}{\chi^{\prime}\left(s_{i}\right)}\right)^{\prime} \mu^{\prime \prime}\left(s_{i}\right) \\
& +\frac{1+\left(\chi\left(s_{i}\right)\right)^{2}}{\chi^{\prime}\left(s_{i}\right)} \mu^{\prime}\left(s_{i}\right) \\
& +\left[\left(\frac{1+\left(\chi\left(s_{i}\right)\right)^{2}}{\chi^{\prime}\left(s_{i}\right)}\right)^{\prime}\right. \\
& \left.+\chi\left(s_{i}\right)\right] \mu\left(s_{i}\right) \mid \cong 0
\end{aligned}
$$

$$
\begin{aligned}
R \delta_{N}\left(s_{i}\right)=\mid \frac{1}{\chi\left(s_{i}\right)} & \delta^{\prime \prime \prime}\left(s_{i}\right)+2\left(\frac{1}{\chi\left(s_{i}\right)}\right)^{\prime} \delta^{\prime \prime}\left(s_{i}\right) \\
& +\left[\left(\frac{1}{\chi\left(s_{i}\right)}\right)^{\prime \prime}\right. \\
& \left.+\frac{1+\left(\chi\left(s_{i}\right)\right)^{2}}{\chi\left(s_{i}\right)}\right] \delta^{\prime}\left(s_{i}\right) \\
& +\chi^{\prime}\left(s_{i}\right) \delta\left(s_{i}\right) \mid \cong 0
\end{aligned}
$$

olup, burada $R \lambda_{N}\left(s_{i}\right) \leq 10^{-k_{i}}, R \mu_{N}\left(s_{i}\right) \leq 10^{-k_{i}}$ ve $R \delta_{N}\left(s_{i}\right) \leq 10^{-k_{i}}\left(k_{i} \in Z^{+}\right)$sağlanmalıdır.

\section{Bulgular}

$\mathrm{Bu}$ bölümde nümerik bir örnek üzerinden eğriyi belirleyen katsayı fonksiyonları sırasıyla Bernstein matris siralama metodu ve Hermite matris siralama metodu ile elde edilmiştir. Ardından elde edilen yaklaşık çözümler için rezidüel fonksiyona dayalı hata analizi yapılarak elde edilen yaklaşık çözümler Tablo 1-9 ve Şekil 1-6 da karşılaştırılmıştır.

Örnek: Öklid uzayında $\rho \tau=s+1$ için $0 \leq s \leq 1$ aralığında sabit genişlikli birim hızlı eğriyi belirleyen katsayı fonksiyonlarını hesaplayıp elde edilen çözümlerin $\lambda_{0}, \mu_{0}, \delta_{0}=1, \lambda_{0}^{\prime}, \mu_{0}^{\prime}, \delta_{0}^{\prime}=0$ ve $\lambda_{0}^{\prime \prime}, \mu_{0}^{\prime \prime}, \delta_{0}^{\prime \prime}=$ 0 için hata analizlerini yapalım. Öncelikle çalışılan aralıkta siralama noktaları $s_{0}=0, s_{1}=\frac{1}{3}, s_{2}=\frac{2}{3}$, $s_{3}=1$ olur ve Bernstein polinom yaklaşımı için

$$
\boldsymbol{D}^{T}=\left[\begin{array}{rrrr}
1 & 0 & 0 & 0 \\
-3 & 3 & 0 & 0 \\
3 & -6 & 3 & 0 \\
-1 & 3 & -3 & 1
\end{array}\right]
$$

olarak hesaplanır. Buradan

$$
\begin{gathered}
w_{00}=P_{0}(0)-\frac{3}{2 \pi} P_{1}(0)+\frac{3}{2 \pi^{2}} P_{2}(0) \\
-\frac{3}{4 \pi^{3}} P_{3}(0), \\
w_{01}=\frac{3}{2 \pi} P_{1}(0)-\frac{3}{\pi^{2}} P_{2}(0)+\frac{9}{4 \pi^{3}} P_{3}(0), \\
w_{02}=\frac{3}{2 \pi^{2}} P_{2}(0)-\frac{9}{4 \pi^{3}} P_{3}(0), \\
w_{03}=\frac{3}{4 \pi^{3}} P_{3}(0)
\end{gathered}
$$

olur. Son olarak $\left(\widetilde{\boldsymbol{W}}_{B}\right)^{-1}$ matrisi

$$
\left[\begin{array}{cccc}
0 & 1 & 0 & 0 \\
0 & 1 & \frac{2 \pi}{3} & 0 \\
0 & 1 & \frac{4 \pi}{3} & \frac{2 \pi^{2}}{3} \\
\frac{1}{w_{03}} & -\frac{w_{00}+w_{01}+w_{02}}{w_{03}} & -\frac{2 \pi\left(w_{01}+2 w_{02}\right)}{3 w_{03}} & -\frac{2 \pi^{2} w_{02}}{3 w_{03}}
\end{array}\right]
$$

şeklinde hesaplanır ve $\boldsymbol{A}=\left(\widetilde{\boldsymbol{W}}_{B}\right)^{-1} \widetilde{\boldsymbol{G}}$ olmak üzere bilinmeyenler matrisi $\mathbf{A}$ için $a_{0}, a_{1}, \ldots, a_{3}$ aşağıdaki gibi bulunur: 


$$
\begin{gathered}
a_{0}=\alpha_{0}, a_{1}=\alpha_{0}+\frac{2 \pi}{3} \alpha_{1} \\
a_{2}=\alpha_{0}+\left(\frac{4 \pi}{3}\right) \alpha_{1}+\left(\frac{2 \pi^{2}}{3}\right) \alpha_{2} \\
a_{3}=\left(-\frac{w_{00}+w_{01}+w_{02}}{w_{03}}\right) \alpha_{0} \\
+\frac{2 \pi\left(w_{01}+2 w_{02}\right)}{3 w_{03}} \alpha_{1} \\
-\left(\frac{2 \pi^{2} w_{02}}{3 w_{03}}\right) \alpha_{2} .
\end{gathered}
$$

Eğer bu $a_{k}$ bilinmeyenleri (9) denkleminde yerine yazılırsa (8) denkleminin Bernstein polinom yaklaşımı ile yaklaşık çözümü

$$
\begin{gathered}
X(s)=a_{0}+\frac{3}{2 \pi}\left(a_{1}-a_{0}\right) s+\frac{3}{4 \pi^{2}}\left(a_{0}-2 a_{1}+\right. \\
\left.a_{2}\right) s^{2}+\frac{1}{8 \pi^{3}}\left(-a_{0}+3 a_{1}-3 a_{2}+a_{3}\right) s^{3}
\end{gathered}
$$

Öyleyse Bernstein polinom yaklaşımı ile eğriyi belirleyen $\lambda(s)$ katsayısı için;

$$
\begin{gathered}
w_{00}=1+\frac{3}{2 \pi}+\frac{3}{\pi^{2}}+\frac{3}{4 \pi^{3}}, \\
w_{01}=-\frac{3}{2 \pi}-\frac{6}{\pi^{2}}-\frac{9}{4 \pi^{3}} \\
w_{02}=\frac{3}{\pi^{2}}+\frac{9}{4 \pi^{3}}, w_{03}=-\frac{3}{4 \pi^{3}} .
\end{gathered}
$$

olarak hesaplanır. Bu durumda

$$
\begin{gathered}
a_{0}=\alpha_{0} \\
a_{1}=\alpha_{0}+\frac{2 \pi}{3} \alpha_{1} \\
a_{2}=\alpha_{0}+\left(\frac{4 \pi}{3}\right) \alpha_{1}+\left(\frac{2 \pi^{2}}{3}\right) \alpha_{2} \\
a_{3}=\frac{4 \pi^{3}+3}{3} \alpha_{0}+\frac{4 \pi^{3}-3 \pi}{3} \alpha_{1} \\
+\frac{8 \pi^{3}+18 \pi^{2}}{3} \alpha_{2}
\end{gathered}
$$

elde edilir. Buradan

$$
\begin{aligned}
\lambda(s)=\alpha_{0}+\alpha_{1} s & +\frac{\alpha_{2}}{2} s^{2}+\left[\frac{1}{6} \alpha_{0}\right. \\
& +\left(\frac{1}{6}-\frac{3}{8 \pi^{2}}\right) \alpha_{1}+\left(\frac{1}{3}\right. \\
& \left.\left.+\frac{1}{2 \pi}\right) \alpha_{2}\right] s^{3}
\end{aligned}
$$

olarak bulunur.

İkinci olarak eğriyi belirleyen $\mu(s)$ katsayısı için;

$$
\begin{gathered}
w_{00}=1+\frac{3}{\pi^{2}}-\frac{9}{4 \pi^{3}}, w_{01}=-\frac{6}{\pi^{2}}+\frac{27}{4 \pi^{3}}, \\
w_{02}=\frac{3}{\pi^{2}}-\frac{27}{4 \pi^{3}}, w_{03}=\frac{9}{4 \pi^{3}}
\end{gathered}
$$

olarak elde edilir. Buradan

$$
\begin{gathered}
a_{0}=\alpha_{0}, a_{1}=\alpha_{0}+\frac{2 \pi}{3} \alpha_{1} \\
a_{2}=\alpha_{0}+\left(\frac{4 \pi}{3}\right) \alpha_{1}+\left(\frac{2 \pi^{2}}{3}\right) \alpha_{2} \\
a_{3}=\frac{9-4 \pi^{3}}{9} \alpha_{0}-2 \pi \alpha_{1}-\frac{8 \pi^{3}-18 \pi^{2}}{9} \alpha_{2}
\end{gathered}
$$

elde edilir. Buradan

$$
\begin{aligned}
\mu(s)=\alpha_{0}+\alpha_{1} s & +\frac{\alpha_{2}}{2} s^{2}+\left[-\frac{1}{18} \alpha_{0}-\frac{1}{2 \pi^{2}} \alpha_{1}\right. \\
& \left.-\frac{1}{9} \alpha_{2}\right] s^{3}
\end{aligned}
$$

olarak bulunur.

Son olarak eğriyi belirleyen $\delta(s)$ katsayısı için;

$$
\begin{gathered}
w_{00}=1+\frac{3}{\pi}+\frac{6}{\pi^{2}}-\frac{3}{4 \pi^{3}} \\
w_{01}=-\frac{3}{\pi}-\frac{12}{\pi^{2}}+\frac{9}{4 \pi^{3}}, w_{02}=\frac{6}{\pi^{2}}-\frac{9}{4 \pi^{3}} \\
w_{03}=\frac{3}{4 \pi^{3}}
\end{gathered}
$$

olarak elde edilir. Buradan

$$
\begin{gathered}
a_{0}=\alpha_{0}, a_{1}=\alpha_{0}+\frac{2 \pi}{3} \alpha_{1} \\
a_{2}=\alpha_{0}+\left(\frac{4 \pi}{3}\right) \alpha_{1}+\left(\frac{2 \pi^{2}}{3}\right) \alpha_{2} \\
a_{3}=\frac{3-4 \pi^{3}}{3} \alpha_{0}-\frac{8 \pi^{3}+6 \pi}{3} \alpha_{1} \\
-\frac{16 \pi^{3}-6 \pi^{2}}{3} \alpha_{2}
\end{gathered}
$$

elde edilir. Buradan

$$
\begin{aligned}
\delta(s)=\alpha_{0}+\alpha_{1} s & +\frac{\alpha_{2}}{2} s^{2}+\left[-\frac{1}{6} \alpha_{0}\right. \\
& \left.-\left(\frac{1}{2 \pi^{2}}+\frac{1}{3}\right) \alpha_{1}-\frac{2}{3} \alpha_{2}\right] s^{3}
\end{aligned}
$$

bulunur.

Hermite polinom yaklaşımı için çalıșılan aralıkta

$$
\begin{gathered}
w_{00}=P_{0}(0), w_{01}=2 P_{1}(0), \\
w_{02}=-2 P_{0}(0)+8 P_{2}(0), \\
w_{03}=-12 P_{1}(0)+48 P_{3}(0)
\end{gathered}
$$

olarak hesaplanır. Buradan $\left(\widetilde{\boldsymbol{W}}_{H}\right)^{-1}$ matrisi

$$
\left[\begin{array}{cccc}
0 & 1 & 0 & \frac{1}{4} \\
\frac{1}{8 P_{3}(0)} & -\frac{P_{0}(0)}{8 P_{3}(0)} & -\frac{P_{1}(0)-4 P_{3}(0)}{8 P_{3}(0)} & -\frac{P_{2}(0)}{8 P_{3}(0)} \\
0 & 0 & 0 & \frac{1}{8} \\
\frac{1}{48 P_{3}(0)} & -\frac{P_{0}(0)}{48 P_{3}(0)} & -\frac{P_{1}(0)}{48 P_{3}(0)} & -\frac{P_{2}(0)}{48 P_{3}(0)}
\end{array}\right]
$$

olmak üzere 


$$
\begin{gathered}
a_{0}=\alpha_{0}+\frac{1}{4} \alpha_{2} \\
a_{1}=-\frac{P_{0}(0)}{8 P_{3}(0)} \alpha_{0}-\frac{P_{1}(0)-4 P_{3}(0)}{8 P_{3}(0)} \alpha_{1}-\frac{P_{2}(0)}{8 P_{3}(0)} \alpha_{2} \\
a_{2}=\frac{1}{8} \alpha_{2} \\
a_{3}=-\frac{P_{0}(0)}{48 P_{3}(0)} \alpha_{0}-\frac{P_{1}(0)}{48 P_{3}(0)} \alpha_{1}-\frac{P_{2}(0)}{48 P_{3}(0)} \alpha_{2}
\end{gathered}
$$

olur. Dolayısıyla yaklaşık çözüm için aşağıdaki eşitlik elde edilir:

$X(s)=a_{0}+2 s a_{1}+\left(4 s^{2}-4\right) a_{2}+\left(8 s^{3}-12 s\right) a_{3}$.

Hermite polinom yaklaşımı ile eğriyi belirleyen $\lambda(s)$ katsayısı için;

$$
\begin{gathered}
w_{00}=P_{0}(0)=1, w_{01}=2 P_{1}(0)=-2, \\
w_{02}=-2 P_{0}(0)+8 P_{2}(0)=14 \\
w_{03}=-12 P_{1}(0)+48 P_{3}(0)=-36
\end{gathered}
$$

olarak hesaplanır. Bu durumda

$$
\begin{gathered}
a_{0}=\alpha_{0}+\frac{1}{4} \alpha_{2}, \\
a_{1}=\frac{1}{8} \alpha_{0}+\frac{3}{8} \alpha_{1}+\frac{1}{24} \alpha_{2} \\
a_{2}=\frac{1}{8} \alpha_{2}, a_{3}=\frac{1}{48} \alpha_{0}-\frac{1}{48} \alpha_{1}+\frac{1}{24} \alpha_{2}
\end{gathered}
$$

olmak üzere

$$
\begin{array}{r}
\lambda(s)=\left(\frac{\alpha_{0}-\alpha_{1}+2 \alpha_{2}}{6}\right) s^{3}+\left(\frac{\alpha_{2}}{2}\right) s^{2} \\
-\left(\frac{5 \alpha_{2}}{12}\right) s+\frac{4 \alpha_{0}+4 \alpha_{1}-\alpha_{2}}{4}
\end{array}
$$

elde edilir.

İkinci olarak eğriyi belirleyen $\mu(s)$ katsayısı için;

$$
\begin{gathered}
w_{00}=P_{0}(0)=3, w_{01}=2 P_{1}(0)=2, \\
w_{02}=-2 P_{0}(0)+8 P_{2}(0)=-6, \\
w_{03}=-12 P_{1}(0)+48 P_{3}(0)=24
\end{gathered}
$$

olarak hesaplanır. Bu durumda

$$
\begin{gathered}
a_{0}=\alpha_{0}+\frac{1}{4} \alpha_{2}, \\
a_{1}=-\frac{1}{2} \alpha_{0}+\frac{1}{3} \alpha_{1}+\frac{1}{12} \alpha_{2}, a_{2}=\frac{1}{8} \alpha_{2} \\
a_{3}=-\frac{1}{12} \alpha_{0}-\frac{1}{36} \alpha_{1}+\frac{1}{72} \alpha_{2}
\end{gathered}
$$

olmak üzere

$$
\begin{gathered}
\mu(s)=\left(\frac{-6 \alpha_{0}-2 \alpha_{1}+\alpha_{2}}{9}\right) s^{3}+\left(\frac{\alpha_{2}}{2}\right) s^{2}+\alpha_{1} s \\
+\frac{4 \alpha_{0}-\alpha_{2}}{4}
\end{gathered}
$$

elde edilir.

Son olarak eğriyi belirleyen $\delta(s)$ katsayısı için;

$$
\begin{gathered}
w_{00}=P_{0}(0)=1, w_{01}=2 P_{1}(0)=8, \\
w_{02}=-2 P_{0}(0)+8 P_{2}(0)=-18, \\
w_{03}=-12 P_{1}(0)+48 P_{3}(0)=0
\end{gathered}
$$

olarak hesaplanır. Bu durumda

$$
\begin{gathered}
a_{0}=\alpha_{0}+\frac{1}{4} \alpha_{2}, a_{1}=\frac{1}{8} \alpha_{0}+\frac{1}{4} \alpha_{2} \\
a_{2}=\frac{1}{8} \alpha_{2}, a_{3}=-\frac{1}{48} \alpha_{0}-\frac{1}{12} \alpha_{1}+\frac{1}{24} \alpha_{2}
\end{gathered}
$$

olmak üzere

$$
\begin{aligned}
\delta(s)=\left(\frac{-\alpha_{0}-4 \alpha_{1}+2 \alpha_{2}}{6}\right) s^{3}+\left(\frac{\alpha_{2}}{2}\right) s^{2} \\
+\left(\frac{\alpha_{0}+2 \alpha_{1}}{2}\right) s+\frac{4 \alpha_{0}-\alpha_{2}}{4}
\end{aligned}
$$

elde edilir.

Tablo 1. $\lambda$ için Bernstein polinomuna dayalı elde edilen yaklașık çözümlerin karșılaștırması

\begin{tabular}{cccc}
\hline $\mathrm{s}$ & $\lambda_{13}(s)$ & $\lambda_{15}(s)$ & $\lambda_{17}(s)$ \\
0.1 & 1.000166490068819 & 1.000166490068963 & 1.000166490068968 \\
0.3 & 1.004452004359640 & 1.004452004361769 & 1.004452004361757 \\
0.5 & 1.020148119614551 & 1.020148119621044 & 1.020148119621049 \\
0.7 & 1.053116717893048 & 1.053116717908712 & 1.053116717890104 \\
0.9 & 1.106061157376765 & 1.106061157441449 & 1.106061157652404 \\
1.0 & 1.139584115444995 & 1.139584116134602 & 1.139584116135300 \\
\hline
\end{tabular}

Tablo 2. $\lambda$ için Hermite polinomuna dayalı elde edilen yaklaşık çözümlerin karşılaștırması

$\begin{array}{cccc}\mathrm{s} & \lambda_{13}(s) & \lambda_{15}(s) & \lambda_{17}(s) \\ 0.1 & 1.000166490068815 & 1.000166490068965 & 1.000166490068968 \\ 0.3 & 1.004452004359631 & 1.004452004361769 & 1.004452004361781 \\ 0.5 & 1.020148119614543 & 1.020148119621044 & 1.020148119621076 \\ 0.7 & 1.053116717892449 & 1.053116717905517 & 1.053116717905579 \\ 0.9 & 1.106061157382274 & 1.106061157419871 & 1.106061157419976 \\ 1.0 & 1.139584115445131 & 1.139584116134653 & 1.139584116135332\end{array}$


Tablo 3. Elde edilen yaklașık çözümler için $R \lambda_{N}(s)$ karșılaștırması

\begin{tabular}{cccc|ccc}
\hline \multicolumn{4}{c}{ Bernstein Matris Siralama Yöntemi } & \multicolumn{2}{c}{ Hermite Matris Siralama Yöntemi } \\
\hline $\mathrm{S}$ & $R \lambda_{13}(s)$ & $R \lambda_{15}(s)$ & $R \lambda_{17}(s)$ & $R \lambda_{13}(s)$ & $R \lambda_{15}(s)$ & $R \lambda_{17}(s)$ \\
0.1 & $2.03018 \mathrm{E}-10$ & $9.67670 \mathrm{E}-13$ & $4.69957 \mathrm{E}-13$ & $2.02204 \mathrm{E}-10$ & $6.66134 \mathrm{E}-13$ & $1.50990 \mathrm{E}-14$ \\
0.3 & $9.95781 \mathrm{E}-12$ & $4.72967 \mathrm{E}-11$ & $4.57957 \mathrm{E}-11$ & $8.04978 \mathrm{E}-12$ & $3.23075 \mathrm{E}-14$ & $1.77636 \mathrm{E}-15$ \\
0.5 & $3.87558 \mathrm{E}-11$ & $1.29896 \mathrm{E}-13$ & $8.78186 \mathrm{E}-14$ & $3.88272 \mathrm{E}-11$ & $2.42029 \mathrm{E}-14$ & $5.77316 \mathrm{E}-15$ \\
0.7 & $1.15543 \mathrm{E}-09$ & $2.90085 \mathrm{E}-10$ & $6.09551 \mathrm{E}-08$ & $2.91565 \mathrm{E}-10$ & $4.91607 \mathrm{E}-13$ & $1.77636 \mathrm{E}-15$ \\
0.9 & $2.00926 \mathrm{E}-06$ & $1.10386 \mathrm{E}-08$ & $3.36753 \mathrm{E}-07$ & $2.00451 \mathrm{E}-06$ & $1.46154 \mathrm{E}-09$ & $2.13672 \mathrm{E}-10$ \\
1.0 & $2.73688 \mathrm{E}-05$ & $2.26618 \mathrm{E}-08$ & $2.52772 \mathrm{E}-08$ & $2.73634 \mathrm{E}-05$ & $1.84410 \mathrm{E}-08$ & $1.21370 \mathrm{E}-08$ \\
\hline
\end{tabular}

Tablo 4. $\mu$ için Bernstein polinomuna dayalı elde edilen yaklaşık çözümlerin karşılaştırması

$\begin{array}{cccc}s & \mu_{13}(s) & \mu_{15}(s) & \mu_{17}(s) \\ 0.1 & 0.9994880476667710 & 0.9994880476661450 & 0.9994880476661320 \\ 0.3 & 0.9856463260229130 & 0.9856463260146350 & 0.9856463260144630 \\ 0.5 & 0.9321003095201960 & 0.9321003094966320 & 0.9321003094961900 \\ 0.7 & 0.8136103905420490 & 0.8136103905005650 & 0.8136103904810700 \\ 0.9 & 0.6143911805978874 & 0.6143911804675874 & 0.6143911806713882 \\ 1.0 & 0.4846460538433713 & 0.4846460500525277 & 0.4846460500124708\end{array}$

Tablo 5. $\mu$ için Hermite polinomuna dayalı elde edilen yaklaşık çözümlerin karşılaştırması

\begin{tabular}{cccc}
$\mathrm{s}$ & $\mu_{13}(s)$ & $\mu_{15}(s)$ & $\mu_{17}(s)$ \\
0.1 & 0.9994880476667740 & 0.9994880476661400 & 0.9994880476661370 \\
0.3 & 0.9856463260229130 & 0.9856463260146330 & 0.9856463260144790 \\
0.5 & 0.9321003095202050 & 0.9321003094966370 & 0.9321003094961840 \\
0.7 & 0.8136103905415000 & 0.8136103904973680 & 0.8136103904965150 \\
0.9 & 0.6143911806033264 & 0.6143911804464828 & 0.6143911804448270 \\
1.0 & 0.4846460538432779 & 0.4846460500524022 & 0.4846460500135315 \\
\hline
\end{tabular}

Tablo 6. Elde edilen yaklaşı çözümler için $R \mu_{N}(s)$ karşılaş̧ırması

\begin{tabular}{cccc|ccc}
\hline \multicolumn{4}{c}{ Bernstein Matris Siralama Yöntemi } & \multicolumn{2}{c}{ Hermite Matris Siralama Yöntemi } \\
\hline $\mathrm{s}$ & $R \mu_{13}(s)$ & $R \mu_{15}(s)$ & $R \mu_{17}(s)$ & $R \mu_{13}(s)$ & $R \mu_{15}(s)$ & $R \mu_{17}(s)$ \\
0.1 & $8.10360 \mathrm{E}-10$ & $1.28466 \mathrm{E}-11$ & $9.09495 \mathrm{E}-13$ & $8.11464 \mathrm{E}-10$ & $1.20437 \mathrm{E}-11$ & $1.42109 \mathrm{E}-14$ \\
0.3 & $4.27391 \mathrm{E}-11$ & $3.72680 \mathrm{E}-11$ & $4.19220 \mathrm{E}-11$ & $2.94009 \mathrm{E}-11$ & $5.63105 \mathrm{E}-13$ & $1.42109 \mathrm{E}-14$ \\
0.5 & $1.31443 \mathrm{E}-10$ & $5.50671 \mathrm{E}-13$ & $1.13243 \mathrm{E}-13$ & $1.31370 \mathrm{E}-10$ & $5.40012 \mathrm{E}-13$ & $1.42109 \mathrm{E}-14$ \\
0.7 & $5.10354 \mathrm{E}-10$ & $3.01497 \mathrm{E}-10$ & $3.92549 \mathrm{E}-08$ & $9.25679 \mathrm{E}-10$ & $1.09921 \mathrm{E}-11$ & $5.68434 \mathrm{E}-14$ \\
0.9 & $6.02981 \mathrm{E}-06$ & $4.77932 \mathrm{E}-08$ & $1.47699 \mathrm{E}-07$ & $6.03200 \mathrm{E}-06$ & $5.37485 \mathrm{E}-08$ & $1.27704 \mathrm{E}-09$ \\
1.0 & $8.04150 \mathrm{E}-05$ & $1.30433 \mathrm{E}-06$ & $1.26919 \mathrm{E}-07$ & $8.04130 \mathrm{E}-05$ & $1.29934 \mathrm{E}-06$ & $6.73749 \mathrm{E}-08$ \\
\hline
\end{tabular}

Tablo 7. $\delta$ için Bernstein polinomuna dayalı elde edilen yaklașık çözümlerin karșılaștırması

\begin{tabular}{cccc}
$\mathrm{S}$ & $\delta_{13}(s)$ & $\delta_{15}(s)$ & $\delta_{17}(s)$ \\
0.1 & 0.9998210246717420 & 0.9998210246715190 & 0.9998210246715120 \\
0.3 & 0.9945475499524850 & 0.9945475499492460 & 0.9945475499490990 \\
0.5 & 0.9723269160326030 & 0.9723269160223580 & 0.9723269160219400 \\
0.7 & 0.9192544538998090 & 0.9192544538812280 & 0.9192544538617170 \\
0.9 & 0.8236221932012510 & 0.8236221931569603 & 0.8236221933633030 \\
1.0 & 0.7582172732955862 & 0.7582172717522533 & 0.7582172717231838 \\
\hline
\end{tabular}

Tablo 8. $\delta$ için Hermite polinomuna dayalı elde edilen yaklaşık çözümlerin karşslaştırması

$\begin{array}{cccc}\mathrm{s} & \delta_{13}(s) & \delta_{15}(s) & \delta_{17}(s) \\ 0.1 & 0.999821024671739 & 0.9998210246715220 & 0.9998210246715130 \\ 0.3 & 0.994547549952478 & 0.9945475499492510 & 0.9945475499491170 \\ 0.5 & 0.972326916032599 & 0.9723269160223710 & 0.9723269160219460 \\ 0.7 & 0.919254453899229 & 0.9192544538780470 & 0.9192544538771680 \\ 0.9 & 0.823622193206711 & 0.8236221931356650 & 0.8236221931339450 \\ 1.0 & 0.758217273295540 & 0.7582172717524069 & 0.7582172717235805\end{array}$


Tablo 9. Elde edilen yaklaşık çözümler için $R \delta_{N}(s)$ karşılaştırması

\begin{tabular}{cccc|ccc}
\hline \multicolumn{4}{c}{ Bernstein Matris Siralama Yöntemi } & \multicolumn{2}{c}{ Hermite Matris Srralama Yöntemi } \\
\hline $\mathrm{s}$ & $R \delta_{13}(s)$ & $R \delta_{15}(s)$ & $R \delta_{17}(s)$ & $R \delta_{13}(s)$ & $R \delta_{15}(s)$ & $R \delta_{17}(s)$ \\
0.1 & $2.52591 \mathrm{E}-10$ & $8.57336 \mathrm{E}-12$ & $4.38427 \mathrm{E}-13$ & $2.52514 \mathrm{E}-10$ & $7.60192 \mathrm{E}-12$ & $4.72955 \mathrm{E}-14$ \\
0.3 & $1.99299 \mathrm{E}-11$ & $2.70088 \mathrm{E}-11$ & $2.19261 \mathrm{E}-11$ & $8.04268 \mathrm{E}-12$ & $2.95430 \mathrm{E}-13$ & $3.99680 \mathrm{E}-15$ \\
0.5 & $1.04964 \mathrm{E}-10$ & $2.23654 \mathrm{E}-13$ & $2.99760 \mathrm{E}-14$ & $3.23106 \mathrm{E}-11$ & $2.36922 \mathrm{E}-13$ & $2.22045 \mathrm{E}-16$ \\
0.7 & $1.04443 \mathrm{E}-09$ & $7.24928 \mathrm{E}-11$ & $1.86078 \mathrm{E}-08$ & $2.07932 \mathrm{E}-10$ & $4.50318 \mathrm{E}-12$ & $1.22125 \mathrm{E}-15$ \\
0.9 & $1.24812 \mathrm{E}-06$ & $1.85438 \mathrm{E}-08$ & $1.27728 \mathrm{E}-07$ & $1.25163 \mathrm{E}-06$ & $2.01511 \mathrm{E}-08$ & $2.55566 \mathrm{E}-10$ \\
1.0 & $1.60844 \mathrm{E}-05$ & $4.68993 \mathrm{E}-07$ & $2.32415 \mathrm{E}-08$ & $1.60908 \mathrm{E}-05$ & $4.70542 \mathrm{E}-07$ & $1.34622 \mathrm{E}-08$ \\
\hline
\end{tabular}

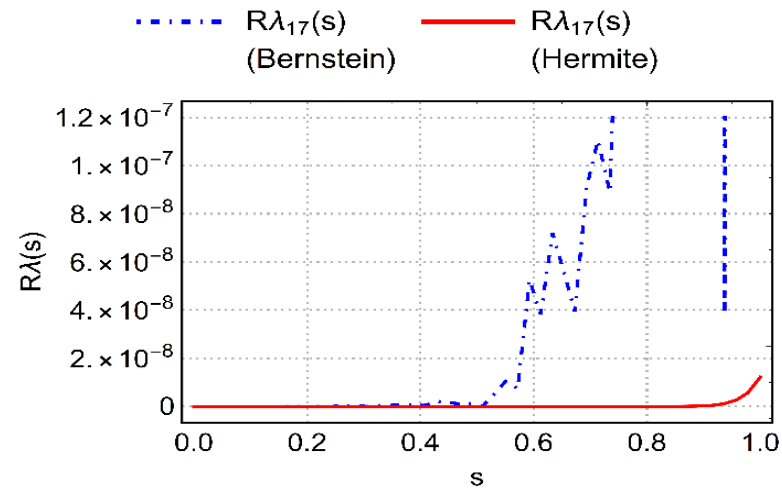

Şekil 1. $\lambda$ katsayı fonksiyonunun $N=17$ için elde edilen yaklaşık çözümlerinin rezidüel hatalarının karşılaştırması
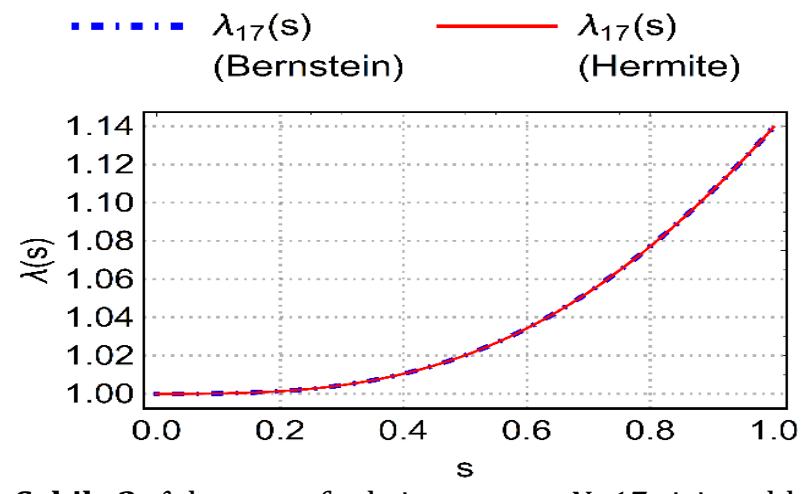

Şekil 2. $\lambda$ katsayı fonksiyonunun $N=17$ için elde edilen yaklaşık çözümlerinin karşılaştırması

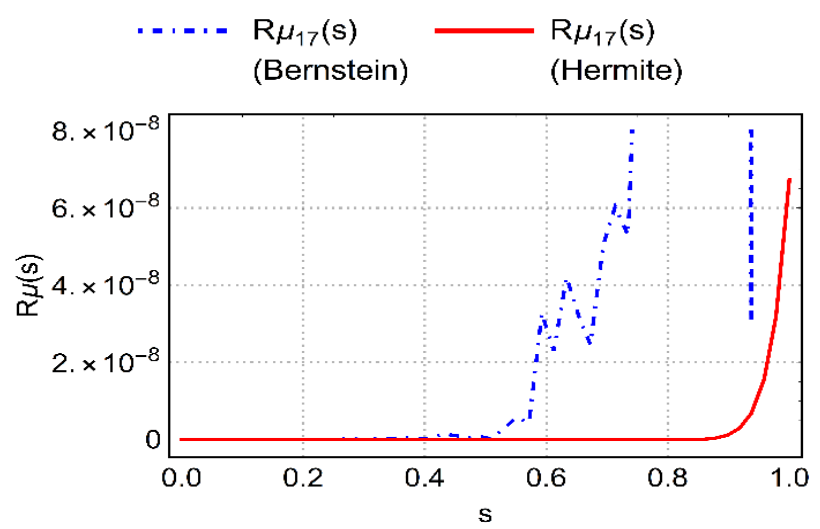

Şekil 3. $\mu$ katsayı fonksiyonunun $N=17$ için elde edilen yaklaşık çözümlerinin rezidüel hatalarının karşılaştırması

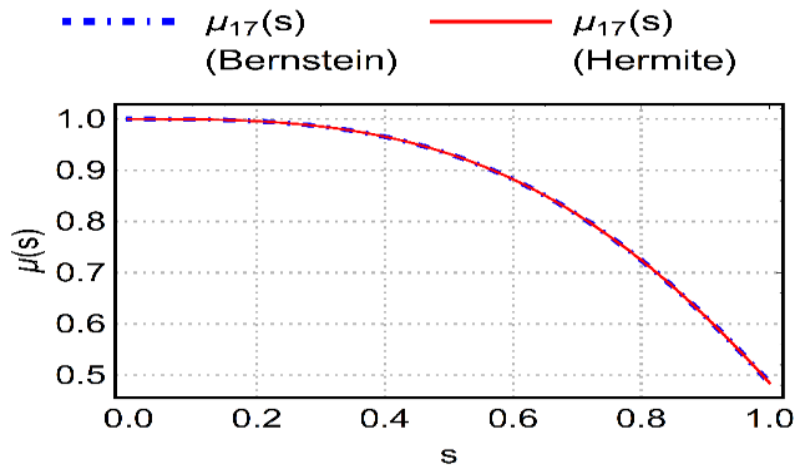

Şekil 4. $\mu$ katsayı fonksiyonunun $N=17$ için elde edilen yaklaşık çözümlerinin karşılaştırması

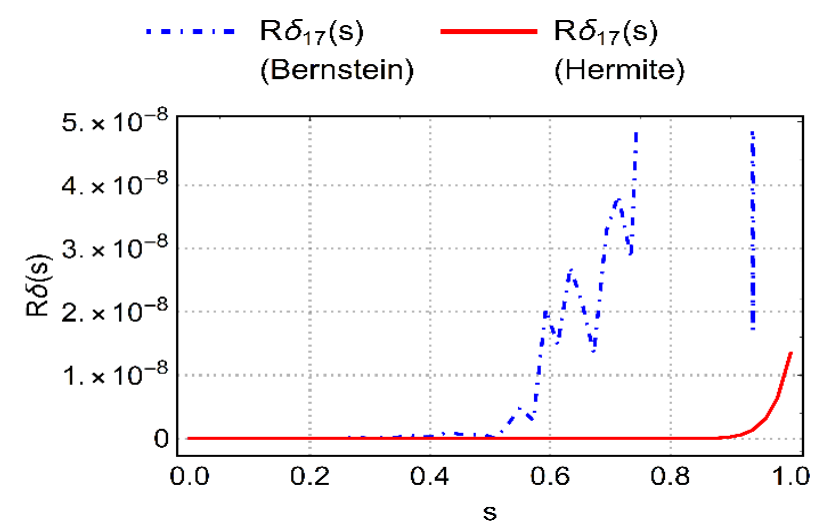

Şekil 5. $\delta$ katsayı fonksiyonunun $N=17$ için elde edilen yaklaşık çözümlerinin rezidüel hatalarının karşılaştırması

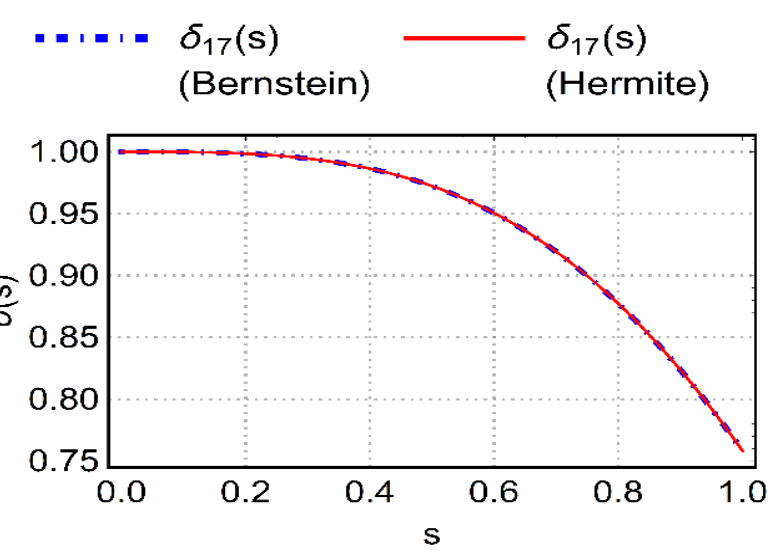

Şekil 6. $\delta$ katsayı fonksiyonunun $N=17$ için elde edilen yaklaşık çözümlerinin karşılaştırması 


\section{Tartışma ve Sonuç}

$\mathrm{Bu}$ makalede öncelikle sabit genişlikli eğriler, eğriyi belirleyen katsayı fonksiyonlarına bağlı üç farklı diferansiyel denklem ile karakterize edilmiştir. Bunun için Frenet benzeri bir diferansiyel denklem sistemi kullanılmıştır. Ardından Hermite ve Bernstein matris sıralama yöntemleri ile bu denklemlerin iki farklı yöntemle yaklaşık çözümleri hesaplanmış ve her bir yöntem için nümerik bir örnek üzerinden hata analizleri yapılmıştır. Buna göre her iki çözüm için de kesme sınırı olan $\mathrm{N}$ değeri arttıkça hata miktarının azaldığ 1 gözlemlenmiştir. Elde edilen tablo ve grafiklerden Hermite polinomuna dayalı yaklaşımın daha iyi sonuçlar verdiği yani daha az hata ile sonuca yaklaştığı gözlemlendi. Dolayısıyla Hermite matris sıralama yönteminin bu eğri tipini karakterize eden denklemler için daha uygun bir yöntem olduğu ifade edilebilir. Sabit genişlikli eğri tipinin diferansiyel denklemlerle ifadesi ve bu yaklaşık çözümler bu eğri tipinin uygulamada daha çok tercih edilmesine imkan tanıyacaktır. Bundan sonra yapılacak çalışmalarda bu eğri tipinin farklı uzaylarda karakterizasyonları elde edilebilir. Farklı uzaylardaki karakterizasyonlar için benzer yöntemlerle yaklaşık çözümler elde edilebilir. Ayrıca sunulan çözüm yöntemleri farklı eğri tiplerinin karakterizasyonları için de kullanılabilir.

\section{Teşekkür}

$\mathrm{Bu}$ çalışma TÜBİTAK 1002 Hızlı Destek Programı 119F213 numaralı Sabit Genişlikli Eğrilerin Analizi ve Uygulama Kapsamının Ön Araştırması başlıklı proje ile desteklenmektedir. Desteğinden dolayı Türkiye Bilimsel ve Teknik Araştırma Kurumu'na teşekkür ederiz.

\section{Etik Beyanı}

Bu çalışmada, "Yükseköğretim Kurumları Bilimsel Araştırma ve Yayın Etiği Yönergesi" kapsamında uyulması gerekli tüm kurallara uyulduğunu, bahsi geçen yönergenin "Bilimsel Araștırma ve Yayın Etiğine Aykırı Eylemler" başlığı altında belirtilen eylemlerden hiçbirinin gerçekleștirilmediğini taahhüt ederiz.

\section{Kaynakça}

[1] Ball, N. H. 1930. On Ovals. American Mathematical Monthly, 27, 348-353.

[2] Köse, Ö. 1986. On Space Curves of Constant Breadth. Doğa Turk Journal of Mathematics. 10(1), 11-14.

[3] Euler, L. 1778-1780. De Curvis Trangularibis. Acta Academica Petropoliteanca. 3-30.

[4] Reuleaux, F. 1963. The Kinematics of Machinery. Trans. By Kennedy A.B.W. Dover Pub. New York.

[5] Mellish, A. P. 1931. Notes of Differential Geometry. Annals of Mathematics, 32, 181-190.
[6] Struik, D. J. 1931. Differential Geometry in the Large. Bulletin American Mathematical Society. 37, 49-62.

[7] Martini, H., Montejano-Peimbert, L., Oliveros, D. 2019. Bodies of Constant Width: An Introduction to Convex Geometry with Applications, Springer Nature Switzerland AG.

[8] Zayas E. E., Cardona S., Jordi L. 2009. Analysis and synthesis of the displacement function of the follower in constant-breadth cam mechanisms. Mechanism and Machine Theory. 44, 1938-1949.

[9] Shchekotov, M. 2015. Indoor Localization Methods Based on Wi-Fi Lateration and Signal Strength Data Collection. Conference of Open Innovation Association (Fruct). 186-191.

[10] Tripathi, A., Gupta, H., Dutta, T., Mishra, R., Shukla, K., Jit, S. 2018. Coverage and Connectivity in WSNs: A Survey. Research Issues and Challenges, IEEE Access, 6, 26971-26992.

[11] Zhu, C., Zheng, C., Shu, L., Han, G. 2012. A Survey on Coverage and Connectivity Issues in Wireless Sensor Networks. Journal Network and Computer Applications. 35, 619-632.

[12] Kolaei, A., Rakheja,S., Richard M.J. 2014. Effects of Tank Cross-section on Dynamic Fluid Slosh Loads and Roll Stability of a Partly-filled Tank Truck. European Journal of Mechanics B/Fluids 46, 46-58.

[13] Altunkaya, B., Aksoyak, F. K. 2017. Curves of Constant Breadth According to Darboux frame, Commun. Fac. Sci. Univ. Ank. Series A1, 66(2), 44-52.

[14] Kocayiğit, H., Önder, M. 2013. Space Curves of Constant Breadth in Minkowski 3-space, Annali di Matematica Pura ed Applicata. 192, 805-814.

[15] Aydın, T. A., Sezer, M. 2018. Hermite Polynomial Approach to Determine Spherical Curves in Euclidean 3-space, New Trends in Mathematical Science, 6(3), 189-199.

[16] Aydın, T. A., Sezer, M., Kocayiğit, H. 2018. Bernsteinn Polynomials Approach to Determine Timelike Curves of Constant Breadth in Minkowski 3-space. Communication in Mathematical Modeling and Applications. 3 (2), 9-22.

[17] Işıı, O. R., Sezer, M., Güney, Z. 2011. A Rational Approximation Based on Bernstein Polynomials for High Order Initial and Boundary Values Problems, Applied Mathematics and Computation, 217, 9438-9450.

[18] Bhatti, M. I., Brocken, B. 2007. Solutions of Differential Equations in A Bernstein Polynomial Basis. Journal of Computational and Applied Mathematics. 205, 272-280. 